\title{
A progressive diagonalization scheme for the Rabi Hamiltonian
}

\author{
Feng Pan, ${ }^{a, b}$ Xin Guan, ${ }^{a}$ Yin Wang, ${ }^{a}$ and J. P. Draayer ${ }^{b}$ \\ ${ }^{a}$ Department of Physics, Liaoning Normal University, Dalian 116029, P. R. China \\ ${ }^{b}$ Department of Physics and Astronomy, Louisiana State University, Baton Rouge, LA \\ 70803-4001, USA
}

\begin{abstract}
A diagonalization scheme for the Rabi Hamiltonian, which describes a qubit interacting with a single-mode radiation field via a dipole interaction, is proposed. It is shown that the Rabi Hamiltonian can be solved almost exactly using a progressive scheme that involves a finite set of one variable polynomial equations. The scheme is especially efficient for the lower part of the spectrum. Some low-lying energy levels of the model with several sets of parameters are calculated and compared to those provided by the recently proposed generalized rotating-wave approximation and a full matrix diagonalization.
\end{abstract}

Keywords: The Rabi Hamiltonian, spin-boson model, progressive diagonalization scheme.

PACS numbers: 42.50.Pq, 42.50.Hz, 85.25.Hv, 02.60.Cb

It is well known that a qubit (two-level atom) interacting with a radiation field is a fundamental problem in modern physics, encountered, for example, in condensed matter, biophysics, and quantum optics. ${ }^{[1,2]}$ The most common approximation assumes that the radiation field is quasi-monochromatic, which leads to the so-called Rabi Hamiltonian. ${ }^{[3,4]}$ Due to its simplicity and importance, the Rabi Hamiltonian has been studied extensively using various methods. More complicated extensions to many two-level atoms interacting with a quasi-mono-chromatic field are described by the Dicke Model. ${ }^{[5]}$ The Rabi Hamiltonian is also equivalent to that of the spin-boson model, ${ }^{[2,6]}$ which describes a qubit interacting with an infinite collection of harmonic oscillators that model the environment acting as a dissipative bosonic bath. The latter model has also attracted considerable attention in various quantum many-body systems due to the rich physics of quantum criticality and decoherence. ${ }^{[7-9]}$ Though it has long been conjectured that the Rabi Hamiltonian may be exactly diagonalizable, ${ }^{[10]}$ with many analytical and numerical studies carried out for decades, ${ }^{[11-21]}$ exact solutions have not been presented except for some special cases. ${ }^{[22-31]}$ Due to the absence of an exact treatment, approximations have been developed. The rotating wave approximation (RWA), ${ }^{[32]}$ which neglects the counter-rotating term in the Hamiltonian, 
a case with the counter-rotating term, and another case with degenerate level of the atom are three well-known solvable cases. ${ }^{[30,31]}$

The Rabi Hamiltonian can be written as ${ }^{[3,4,32]}$

$$
\hat{H}_{\mathrm{R}}=\omega_{0} a^{\dagger} a+\omega \hat{S}_{0}+g\left(a^{\dagger}+a\right)\left(\hat{S}_{-}+\hat{S}_{+}\right)
$$

where $\omega_{0}$ and $\omega$ are frequencies of a single-field mode and the level splitting of the twolevel atom, respectively, $g$ is the coupling parameter between the atom and the field, $a$ and $a^{\dagger}$ are annihilation and creation operators of the field mode, respectively, satisfying the usual bosonic commutation relation: $\left[a, a^{\dagger}\right]=1,[a, a]=\left[a^{\dagger}, a^{\dagger}\right]=0, \hat{S}_{0}$ is the operator of atomic inversion, and $\hat{S}_{+}\left(\hat{S}_{-}\right)$is the atomic raising (lowering) operator, which satisfy the $\mathrm{SU}(2)$ Lie algebraic relations $\left[\hat{S}_{0}, \hat{S}_{ \pm}\right]= \pm \hat{S}_{ \pm},\left[\hat{S}_{+}, \hat{S}_{-}\right]=2 \hat{S}_{0}$. The pseudo-spin operator $\hat{S}^{2}=\hat{S}_{+} \hat{S}_{-}+\hat{S}_{0}\left(\hat{S}_{0}-1\right)$ and the parity $\hat{P}=\exp \left(i \pi\left(a^{\dagger} a+\hat{S}_{0}+S\right)\right)$, where $S=1 / 2$ is the pseudo spin of the atom, commute with the Hamiltonian (1), namely $\left[\hat{S}^{2}, \hat{H}_{\mathrm{R}}\right]=0$ and $\left[\hat{P}, \hat{H}_{\mathrm{R}}\right]=0$.

Let $|0 ; \mu\rangle(\mu=+$ or -$)$ be simultaneously the boson vacuum and the eigenstate of $\hat{S}_{0}$ satisfying $a|0 ; \mu\rangle=0, \hat{S}_{0}|0 ; \pm\rangle= \pm \frac{1}{2}|0 ; \pm\rangle$. The Hamiltonian (1) under the basis spanned by $\left\{|k ; \mu\rangle=\frac{1}{\sqrt{k !}} a^{\dagger k}|0 ; \mu\rangle ; \quad k=0,1,2, \cdots\right\}$ has the following tridiagonal form:

$$
\hat{H}_{\mathrm{R}}=\left(\begin{array}{cc}
H^{(+)} & 0 \\
0 & H^{(-)}
\end{array}\right),
$$

of which the order of the Fock states is $|0 ;+\rangle,|1 ;-\rangle,|2 ;+\rangle, \cdots, \cdots,|0 ;-\rangle,|1 ;+\rangle,|2 ;-\rangle$, $\cdots$, where the two tridiagonal infinite sub-matrices $H^{(+)}$and $H^{(-)}$are given by

$$
\hat{H}^{( \pm)}=\left(\begin{array}{ccccc} 
\pm \frac{1}{2} \omega & g \sqrt{1} & 0 \cdots & & \\
g \sqrt{1} & \omega_{0} \mp \frac{1}{2} \omega & g \sqrt{2} & 0 \cdots & \\
0 & g \sqrt{2} & 2 \omega_{0} \pm \frac{1}{2} \omega & g \sqrt{3} & 0 \cdots \\
\vdots & \ddots & \ddots & \ddots & \ddots
\end{array}\right)
$$

Under the afore arranged order of the basis, the effective Hamiltonian (3) may be written as

$$
\hat{H}^{( \pm)}=\left(\omega_{0} a^{\dagger} a+g\left(a+a^{\dagger}\right)\right) \pm \frac{1}{2} \omega \cos \left(\pi a^{\dagger} a\right) .
$$

The spin component $\mu=+$ or - in the Fock states $|k ; \mu\rangle$ will be omitted in the following for simplicity. The first term in (4) is easily diagonalizable under the shifted boson states 


$$
\mid n)=e^{-\frac{1}{2}\left(g / \omega_{0}\right)^{2}} \sqrt{\frac{1}{n !}}\left(a^{\dagger}+g / \omega_{0}\right)^{n} e^{-\frac{g}{\omega_{0}} a^{\dagger}}|0\rangle
$$

with the corresponding eigenvalues $E_{n}=\omega_{0} n-g^{2} / \omega_{0}$ for $n=0,1,2, \cdots$. The second term is diagonal in the original boson subspace spanned by the Fock states $\{|k\rangle\}$. Therefore, the Hamiltonian (4) can also be expressed as

$$
\left.\hat{H}^{( \pm)}=\sum_{n=0}^{\infty} E_{n} \mid n\right)\left(n\left| \pm \frac{\omega}{2} \mp \omega \sum_{k=0}^{\infty}\right| 2 k+1\right\rangle\langle 2 k+1| .
$$

Since the structure of the Hamiltonians $\hat{H}^{(+)}$and $\hat{H}^{(-)}$is the same, in the following, we take $\hat{H}^{(+)}$to elucidate our diagonalization procedure, and assume that the parameters $\omega_{0}$, $\omega$, and $g$ are all non-zero. We adopt the analytical step-by-step diagonalization procedure proposed in [33]. In the $k$-th step, we take a diagonalized part of the Hamiltonian $\hat{H}^{(k-1)}$ in the shifted boson basis (5) and the $k$-th projection in the second term of (6) with $\hat{H}^{(k)}=$ $\hat{H}^{(k-1)}-\omega|2 k+1\rangle\langle 2 k+1|$ to do the diagonalization. In the initial $k=0$ step,

$$
\left.\hat{H}^{(0)}=\sum_{n=0}^{\infty} E_{n} \mid n\right)\left(n\left|+\frac{\omega}{2}-\omega\right| 1\right\rangle\langle 1|,
$$

which can be diagonalized exactly with the eigenstates

$$
\left.\left|\psi_{\tau_{0}}^{(0)}\right\rangle=\sqrt{\frac{1}{N_{\tau_{0}}^{(0)}}} \sum_{n=0}^{\infty} \frac{\alpha_{n}^{1}}{E_{n}+\omega / 2-E_{\tau_{0}}^{(0)}} \mid n\right),
$$

where $N_{\tau_{0}}^{(0)}$ is the normalization constant, $\alpha_{n}^{2 k+1}$ is the overlap of the original Fock state $|2 k+1\rangle$ with the shifted state $\mid n)$ given by

$$
\alpha_{n}^{2 k+1}=\left(n|2 k+1\rangle=\sqrt{(2 k+1) ! n ! e^{-\left(\frac{g}{\omega_{0}}\right)^{2}}}\left(\frac{g}{\omega_{0}}\right)^{n-2 k-1} \sum_{q} \frac{(-)^{q}\left(g / \omega_{0}\right)^{2 q}}{(n-2 k-1+q) !(2 k+1-q) ! q !} .\right.
$$

One can directly check with the eigenequation $\hat{H}^{(0)}\left|\psi_{\tau_{0}}^{(0)}\right\rangle=E_{\tau_{0}}^{(0)}\left|\psi_{\tau_{0}}^{(0)}\right\rangle$ that (8) is indeed the eigenstate of $\hat{H}^{(0)}$ with the corresponding eigenvalue $E_{\tau_{0}}^{(0)}$ if the following equation is satisfied:

$$
1-\omega \sum_{n=0}^{\infty} \frac{\left(\alpha_{n}^{1}\right)^{2}}{E_{n}+\omega / 2-E_{\tau_{0}}^{(0)}}=0
$$

where $\tau_{0}$ labels the $\tau_{0}$-th root of Eq. (10). However, as shown in Fig. 1, the overlaps $\alpha_{n}^{2 k+1}$ with fixed $k$ are only non-negligible for a finite number of values $n$ with $n=m_{1}, m_{1}+$ $1, \cdots, m_{2}$. As a result, only a finite number of states $\left.\{\mid n)\right\}$ correlate among each other in 
the $k$-th step, which, in turn, effectively truncates the infinite sum in (10) into a finite sum of those terms with non-negligible $\alpha_{n}^{2 k+1}$ for $n=m_{1}, m_{1}+1, \cdots, m_{2}$. The number of values $n$ defined by $d_{k}=m_{2}-m_{1}+1$ is called correlation length. While other states with $n>m_{2}$ or $<m_{1}$ remain essentially unchanged after the diagonalization. The correlation length $d_{k}$ increases with increasing $k$ and the coupling parameter $g / \omega_{0}$. Typically, $d_{k} \sim 10-20$ when $g / \omega_{0}<1$, and $d_{k} \sim 20-60$ when $1<g / \omega_{0}<2$. Furthermore, only those states $\left.\{\mid n)\right\}$ within the correlated region will be correlated among each other. In short, the matrix form of Hamiltonian $\hat{H}^{(k)}$ in the shifted boson basis is almost block diagonal. As a consequence, Eq. (10) can effectively be written as

$$
1-\omega \sum_{n=0}^{d_{0}-1} \frac{\left(\alpha_{n}^{1}\right)^{2}}{E_{n}+\omega / 2-E_{\tau_{0}}^{(0)}}=0,
$$

where $m_{1}=0$ and $m_{2}=d_{0}-1$. After the initial step, $\hat{H}^{(+)}$given by (6) can be rewritten as

$$
\hat{H}^{(+)}=\left(\sum_{\tau_{0}=0}^{d_{0}-1} E_{\tau_{0}}^{(0)}\left|\psi_{\tau_{0}}^{(0)}\right\rangle\left\langle\psi_{\tau_{0}}^{(0)}\left|+\sum_{n=d_{0}+1}^{\infty} E_{n}\right| n\right)(n \mid)-\omega \sum_{k=1}^{\infty}|2 k+1\rangle\langle 2 k+1|,\right.
$$
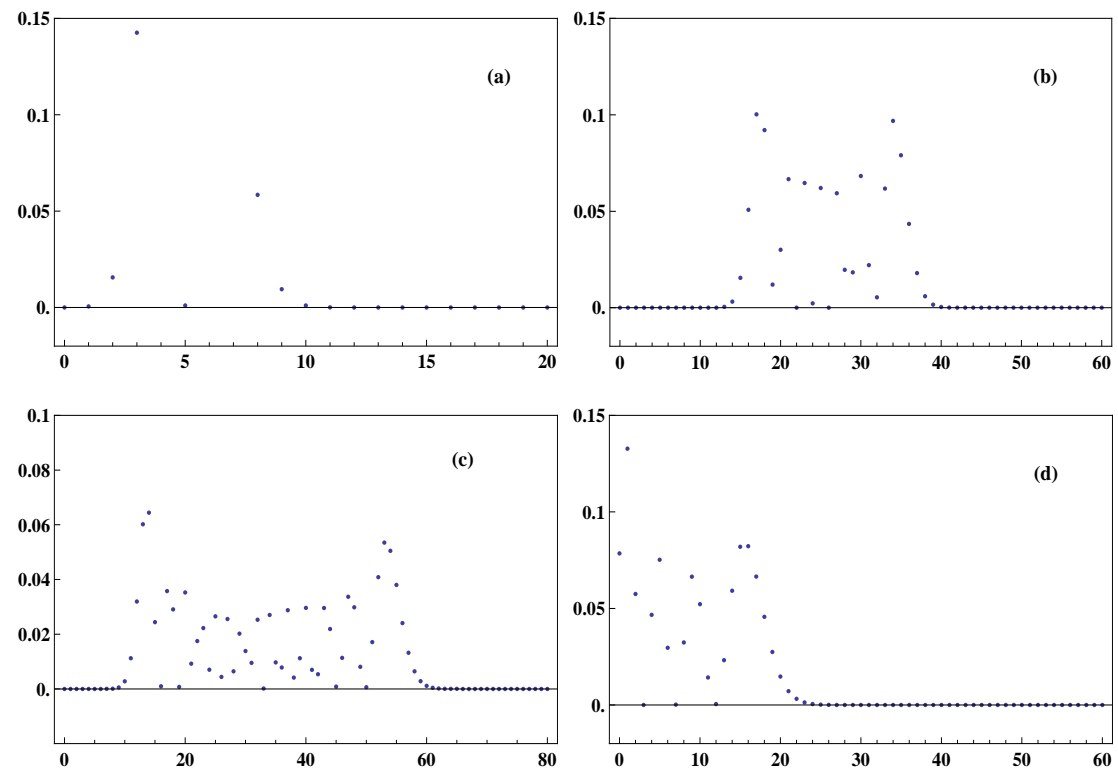

Fig. 1. Overlaps $\alpha_{n}^{2 k+1}$ as functions of $n$ for several values of $k$ and the coupling parameters $g / \omega_{0}$. (a) $k=2, g / \omega_{0}=0.5$; (b) $k=12, g / \omega_{0}=1.0$; (c) $k=15, g / \omega_{0}=2.0$; (d) $k=3, g / \omega_{0}=2.5$. It can be seen that the corresponding correlation length is (a) $d_{2} \sim 10$, (b) $d_{12} \sim 30$, (c) $d_{15} \sim 50$, and $(\mathrm{d}) d_{1} \sim 25$, respectively. 
where

$$
\left.\left|\psi_{\tau_{0}}^{(0)}\right\rangle=\sqrt{\frac{1}{N_{\tau_{0}}^{(0)}}} \sum_{n=0}^{d_{0}-1} \frac{\alpha_{n}^{1}}{E_{n}+\omega / 2-E_{\tau_{0}}^{(0)}} \mid n\right) .
$$

Next, using a similar procedure, the first term and the $k=1$ projection in the second term of (12) given by

$$
\hat{H}^{(1)}=\sum_{\tau_{0}=0}^{d_{0}-1} E_{\tau_{0}}^{(0)}\left|\psi_{\tau_{0}}^{(0)}\right\rangle\left\langle\psi_{\tau_{0}}^{(0)}\left|+\sum_{n=d_{0}}^{\infty} E_{n}\right| n\right)(n|-\omega| 3\rangle\langle 3|
$$

needs to be diagonalized. If the correlation length $d_{1}>d_{0}$, the correlated eigenstates may be written as

$$
\left.\left|\psi_{\tau_{1}}^{(1)}\right\rangle=\sqrt{\frac{1}{N_{\tau_{1}}^{(1)}}}\left(\sum_{\tau_{0}=l}^{d_{0}-1} \frac{\left\langle\psi_{\tau_{0}}^{(0)} \mid 3\right\rangle}{E_{\tau_{0}}^{(0)}-E_{\tau_{1}}^{(1)}}\left|\psi_{\tau_{0}}^{(0)}\right\rangle+\sum_{n=d_{0}}^{d_{1}-1} \frac{\alpha_{n}^{3}}{E_{n}+\omega / 2-E_{\tau_{1}}^{(1)}} \mid n\right)\right),
$$

where it is assumed that $\left\langle\psi_{\tau_{0}}^{(0)} \mid 3\right\rangle \sim 0$ when $\tau_{0}<l$, and

$$
\left\langle\psi_{\tau_{0}}^{(0)} \mid 3\right\rangle=\sqrt{\frac{1}{N_{\tau_{0}}^{(0)}}} \sum_{n=0}^{d_{0}-1} \frac{\alpha_{n}^{1} \alpha_{n}^{3}}{E_{n}+\omega / 2-E_{\tau_{0}}^{(0)}}
$$

according to (13), while the uncorrelated eigenstates satisfy $\left|\psi_{\tau}^{(1)}\right\rangle=\left|\psi_{\tau}^{(0)}\right\rangle$ with the corresponding eigenvalue $E_{\tau}^{(0)}$ for $\tau\left\langle l\right.$, and $\left.\mid \psi_{n}^{(1)}\right\rangle=\mid n$ ) with the corresponding eigenvalue $E_{n}$ for $n \geq d_{1}$. Using (14) and (15) to solve the eigenequation $\hat{H}^{(1)}\left|\psi_{\tau_{1}}^{(1)}\right\rangle=E_{\tau_{1}}^{(1)}\left|\psi_{\tau_{1}}^{(1)}\right\rangle$, one can prove that $(15)$ is indeed the eigenstate of $\hat{H}^{(1)}$ if the corresponding eigenvalue $E_{\tau_{1}}^{(1)}$ is the $\tau_{1}$-th root of the following equation:

$$
1-\omega\left(\sum_{\tau_{0}=l}^{d_{0}-1} \frac{\left(\left\langle\psi_{\tau_{0}}^{(0)} \mid 3\right\rangle\right)^{2}}{E_{\tau_{0}}^{(0)}-E_{\tau_{1}}^{(1)}}+\sum_{n=d_{0}}^{d_{1}-1} \frac{\left(\alpha_{n}^{3}\right)^{2}}{E_{n}+\omega / 2-E_{\tau_{1}}^{(1)}}\right)=0 .
$$

According to this procedure, once eigenvectors $\left|\psi_{\tau_{k}}^{(k)}\right\rangle$ and the corresponding eigenvalues $E_{\tau_{k}}^{(k)}$ are known from the $k$-th step, the $k+1$-th step results are given by

$$
\left|\psi_{\tau_{k+1}}^{(k+1)}\right\rangle=\sqrt{\frac{1}{N_{\tau_{k+1}}^{(k+1)}}} \sum_{\tau_{k}=m_{1}}^{m_{2}} \frac{\left\langle\psi_{\tau_{k}}^{(k)} \mid 2 k+1\right\rangle}{E_{\tau_{k}}^{(k)}-E_{\tau_{k+1}}^{(k+1)}}\left|\psi_{\tau_{k}}^{(k)}\right\rangle
$$

for $\tau_{k+1}=m_{1}, m_{1}+1, \cdots, m_{2}$ and $d_{k+1}=m_{2}-m_{1}+1$, in which $E_{\tau_{k+1}}^{(k+1)}$ should satisfy

$$
1-\omega \sum_{\tau_{k}=m_{1}}^{m_{2}} \frac{\left(\left\langle\psi_{\tau_{k}}^{(k)} \mid 2 k+1\right\rangle\right)^{2}}{E_{\tau_{k}}^{(k)}-E_{\tau_{k+1}}^{(k+1)}}=0 .
$$

While $\left|\psi_{\tau_{k+1}}^{(k+1)}\right\rangle=\left|\psi_{\tau_{k+1}}^{(k)}\right\rangle$ with the corresponding eigenvalue $E_{\tau_{k+1}}^{(k+1)}=E_{\tau_{k+1}}^{(k)}$ for $\tau_{k+1}=$ $0,1, \cdots, m_{1}-1$, and $\tau_{k+1}=m_{2}+1, m_{2}+2, \cdots$. In fact, with increasing $k$, the region 
TABLE I: The first few low-lying energy levels (ordered from the lowest) determined by the Rabi Hamiltonian (1) obtained from the Progressive Diagonalization Scheme (PDS) are compared to those calculated using the GRWA and results from a Full Matrix Diagonalization (FMD), which is done by diagonalizing the infinite tridiagonal matrix (3) in a truncated Hilbert subspace.

\begin{tabular}{ccccccccc}
\hline \hline \multicolumn{3}{c}{$g / \omega_{0}=0.5, \omega_{0}=\omega$} & \multicolumn{2}{c}{$g / \omega_{0}=1.0, \omega_{0}=\omega$} & \multicolumn{3}{c}{$g / \omega_{0}=2.0, \omega_{0}=0.75 \omega$} \\
\hline PDS & GRWA & FMD & PDS & GRWA & FMD & PDS & GRWA & FMD \\
\hline-0.6332 & -0.5532 & -0.6333 & -1.1479 & -1.0677 & -1.1479 & -3.0225 & -3.0002 & -3.0225 \\
-0.1200 & -0.0602 & -0.1200 & -1.0100 & -0.9936 & -1.0102 & -3.0221 & -2.9999 & -3.0222 \\
0.6953 & 0.7401 & 0.6954 & -0.2317 & -0.2110 & -0.2317 & -2.2793 & -2.2546 & -2.2794 \\
0.8253 & 0.8635 & 0.8253 & 0.1334 & 0.2642 & 0.1334 & -2.2738 & -2.2474 & -2.2739 \\
1.5870 & 1.5977 & 1.5870 & 0.9270 & 0.9403 & 0.9270 & -1.5524 & -1.5142 & -1.5525 \\
1.9355 & 1.9115 & 1.9355 & 1.1048 & 1.0539 & 1.1048 & -1.5172 & -1.4966 & -1.5172 \\
2.5485 & 2.5375 & 2.5485 & 1.8428 & 1.8559 & 1.8429 & -0.8554 & -0.8385 & -0.8554 \\
2.9477 & 2.9528 & 2.9478 & 2.1436 & 2.1269 & 2.1437 & -0.7376 & -0.6792 & -0.7376 \\
\hline \hline
\end{tabular}

of the correlation among $\{\mid n)\}$ moves to larger values of $n$, and keeps lower part of the levels with small $n$ less affected. Therefore, after a number of steps, several of the low-lying eigenstates of the Hamiltonian (6) and the corresponding eigenvalues can be obtained. The results are almost exact because the overlaps $\left\langle\psi_{\tau_{k}}^{(k)} \mid 2 k+1\right\rangle$ are small enough to be neglected when $\tau_{k}$ satisfies $\left\langle\psi_{\tau_{k}}^{(k)} \mid 2 k+1\right\rangle \sim 0$, which is similar to the situations shown in Fig. 1 for $\alpha_{n}^{2 k+1}=\left(n|2 k+1\rangle\right.$ because the overlaps $\left\langle\psi_{\tau_{k}}^{(k)} \mid 2 k+1\right\rangle$ is a linear function of $\alpha_{n}^{2 k+1}=(n|2 k+1\rangle$.

Since the Rabi Hamiltonian can not be solved exactly except the case with the RWA ${ }^{[32]}$ and other two special cases studied in [30-31]. As shown in [20], the Generalized RotatingWave Approximation (GRWA) is significantly more accurate than the RWA. To demonstrate our Progressive Diagonalization Scheme (PDS), a few low-lying energy levels with three sets of model parameters are calculated and compared to those calculated using the GRWA and a Full Matrix Diagonalization (FMD), which is done by diagonalizing the infinite tridiagonal matrix (3) in a truncated Hilbert subspace. The dimension of the truncated subspace, with which the first few low-lying energies keep unchanged with further increasing of the dimension, depends on the coupling parameter $g / \omega_{0}$. For $g / \omega_{0}=0.5-2.0$, the dimension of the truncated subspace is $10^{3}-10^{5}$, with which the FMD can be preformed on a PC. When 
$g / \omega_{0}>2.0$, in order to get accurate results, the FMD needs more computer memory to store matrix elements and many hours of CPU time to do the diagonalization. Therefore, we only compare the PDS with the GRWA and the FMD for $g / \omega_{0} \leq 2.0$. The results are shown in Table 1. The PDS results were obtained with less then 4 steps for $g / \omega_{0}=0.5,14$ steps for $g / \omega_{0}=1.0$, and less than 30 steps for $g / \omega_{0}=2.0$, which are exactly the same as the corresponding results obtained from a full matrix diagonalization. In each step, only a single variable finite order polynomial equation needs to be solved. The order of the polynomial equations used in the PDS depends on the correlation length $d_{k}$. In the cases shown in Table 1 , the maximal $d_{k}$ is around 60 when $g / \omega_{0}=2.0$. Such polynomial equations can easily be solved by using Mathematica with a few seconds of CPU time. Though the PDS for $g / \omega_{0}=2.0$ needs about 30 steps in order to get accurate results, the total CPU time needed is much less than that of the FMD. Besides the FMD needs more memory, the CPU time needed is 10-20 times more than the total CPU time needed for the PDS. The PDS results are certainly better than those obtained from the GRWA, which nonetheless has the advantage of being a simpler to implement.

In conclusion, a diagonalization scheme of the Rabi Hamiltonian in the shifted boson basis is proposed based on the step-by step diagonalization method, from which lower part of eigenstates and the corresponding eigenvalues can be obtained progressively within a finite number of steps. In each step, only a single variable finite order polynomial equation needs to be solved due to the fact that only a finite number of the shifted boson states correlate among each other. Such type of polynomial equations also appear in the Tamm-Dancoff and random phase approximations with separable potentials in nuclear physics. ${ }^{[34]}$ The convergence is related to the number of steps that are needed, which in turn depends on the coupling parameter $g / \omega_{0}$ and and position of the energy levels to be calculated. Only a few steps are needed for $g / \omega_{0}<1$, while 10-30 steps are needed in order to get accurate results for the low-lying part of the spectrum for $1<g / \omega_{0} \leq 2$. More steps are needed for higher excited states. Anyway, the total CPU time needed is much less than that of the truncated matrix diagonalization for the low-lying part of the spectrum. As shown in [20], the coupling $g / \omega_{0}$ is often small with $g / \omega_{0}<1$ in quantum optics, microwave resonator, superconducting LC circuits, but $g / \omega_{0} \sim 1$ for nano-mechanical resonator coupled to a charged qubit. Higher values of $g / \omega_{0}$ may also be possible for spin-boson models of other systems. Since the PDS scheme yields nearly exact results that seem to be independent of the model parameters $\omega_{0}, g$, and $\omega$, and the single variable finite order polynomial equation involved in each step is much more easily to be solved than the truncated matrix diagonalization, it should be a useful 
scheme for studying dynamics in the spin-boson model for strong coupling cases, especially

in studying quantum critical phenomena and decoherence, where accurate solutions to the problem is essential. Related work is in progress.

Support from the U.S. National Science Foundation (PHY-0500291 \& OCI-0904874), the Southeastern Universities Research Association, the Natural Science Foundation of China (10775064), the Liaoning Education Department Fund (2007R28), and the LSU-LNNU joint research program (9961) is acknowledged.

\section{References}

[1] L. Allen and J. H. Eberly, Optical Resonance and Two-Level Atoms (Wiley, New York, 1975).

[2] A. J. Leggett, S. Chakravarty, A. T. Dorsey, M. P. A. Fisher, A. Garg, and W. Zwerger, Rev. Mod. Phys. 59, 1 (1987).

[3] I. I. Rabi, Phys. Rev. 49, 324 (1926).

[4] I. I. Rabi, Phys. Rev 51, 652 (1937).

[5] R. Dicke, Phys. Rev. 93, 99 (1954).

[6] M. Blume, V. J. Emery, and A. Luther, Phys. Rev. Lett. 25, 450 (1970).

[7] K. L. Hur, P. D. Beaupré, and W. Hofstetter, Phys. Rev. Lett. 99, 126801(2007).

[8] A. Kopp and K. L. Hur, Phys. Rev. Lett. 98, 220401(2007).

[9] U. Weiss, Quantum Dissipative Systems (World Scientific, Singapore, 1993).

[10] H. G. Reik and M. Doucha, Phys. Rev. Lett. 57, 787 (1986).

[11] T. Yabuzaki, S. Nakayama, Y. Murakami, and T. Ogawa, Phys. Rev. A 10, 1955 (1974).

[12] M. Kus, Phys. Rev. Lett. 54, 1343 (1985).

[13] L. Müller, J. Stolze, H. Leschke, and P. Nagel, Phys. Rev. A 44, 1022 (1991).

[14] L. Bonci, R. Roncaglia, B. J. West, and P. Grigolini, Phys. Rev. Lett. 67, 2593 (1991).

[15] L. Bonci and P. Grigolini, Phys. Rev. A 46, 4445 (1992).

[16] R. F. Bishop, N. J. Davidson, R. M. Quick, and D. M. van der Walt, Phys. Rev. A 54, R4657 (1996).

[17] A. Pereverzev and E. R. Bittner, Phys. Chem. Chem. Phys. 8, 1378 (2006).

[18] V. Fessatidis, J. D. Mancini, S. P. Bowenb, Phys. Lett. A 297, 100 (2002).

[19] R. Graham and M. Höhnerbach, Z. Phys. B 57, 233 (1984).

[20] E. K. Irish, Phys. Rev. Lett. 99, 173601 (2007); Phys, Rev. Lett. 99, 259901 (E) (2007).

[21] T. Liu, K. L. Wang and M. Feng, EPL 86, 54003 (2009). 
[22] V. Loorits, J. Phys C 16, L711 (1983).

[23] M. Kus and M. Lewenstein, J. Phys. A 19, 305 (1986).

[24] R. Koç, Hütüncüler, and M. Koca, J. Phys. A 35, 9425 (2002).

[25] R. F. Bishop, N. J. Davidson, R. M. Quick, and D. M. van der Walt, Phys. Rev. A 54, R4657 (1996).

[26] M. Szopa, G. Mys, A. Ceulemans, J. Math. Phys. 37, 5402 (1996).

[27] C. Emary and R. F. Bishop, J. Math. Phys. 43, 3916 (2002).

[28] S. N. Dolya and O. B. Zaslavskii, J. Phys. A 33, L369 (2000).

[29] S. N. Dolya and O. B. Zaslavskii, J. Phys. A 345955 (2001).

[30] Feng Pan, Y.-K. Yao, M.-X. Xie, W.-J. Han, and J. P. Draayer, Commun. Theor. Phys. 48, 53 (2007).

[31] E. K. Irish, J. Gea-Banacloche, I. Martin, and K. C. Schwab, Phys. Rev. B 72, 195410 (2005).

[32] E. T. Jaynes and F. W. Cummings, Proc. IEEE 51, 89 (1963).

[33] Feng Pan, Ming-Xia Xie, Xin Guan, Lian-Rong Dai, and J. P. Draayer, Phys. Rev. C 80, 044306 (2009).

[34] P. Ring and P. Schuck, The Nuclear Many-Body Problem (Springer-Verlag, Berlin, 1980). 